\title{
PPY-CHOLIC ACID COMPOSITE FOR ETHANOL SENSING THROUGH IMPEDANCE SPECTROSCOPY MEASUREMENT
}

\author{
Partha P. D. Sharma ${ }^{1}$, D. Sarkar ${ }^{2}$ \\ ${ }^{1}$ Department of Physics, Gauhati University, Guwahati, Assam, India \\ ${ }^{2}$ Department of Physics, Gauhati University, Guwahati, Assam, India
}

\begin{abstract}
Polymerization of pyrrole with cholic acid has been achieved by its oxidative polymerization in presence of dodecyl benzenesulphonic acid (DBSA). Incorporation of cholic acid in the composite is confirmed by SEM and FTIR results. The change is indicated by SEM and FTIR spectroscopy. The composite is used for sensing ethanol vapour which is visualized through change in electrical conductivity with applied dc bias 0-1V and low frequency ac bias of 0-5V. After ethanol exposure, resistivity of the composite is found to change from 3.03 to $0.71 \mathrm{Ohmcm}$. Low frequency dispersion is more prominently observed in the ethanol exposed composite, with a linear pattern at $50 \mathrm{~Hz}$ which gradually shows a non-linear trend with increasing frequency. Impedance shows a decreasing trend with increasing frequency on exposure to ethanol below the frequency of $500 \mathrm{~Hz}$; but shows a completely different nature at applied frequency of $500 \mathrm{~Hz}$. Impedance is also found to decrease with increasing exposure time upto 10 minutes, beyond which change in impedance of the ethanol exposed composite is not so prominent. Specific interaction existing between PPy and cholic acid molecule in the composite, which may have directional property as well as the selective binding with the host molecule, are supposed to form the complimentary bonds between them which might become stronger with exposure to ethanol. The polymer composite also undergoes a chemical reaction on exposure to ethanol vapour. Non-covalent interaction takes place between the aromatic rings of the polymer composite and the ionic species of oxygen, give rise to ' $\pi$ stacking' which eventually leads to changing electronic conductivity in terms of sensing behavior.
\end{abstract}

\section{INTRODUCTION}

Bile acid, which is molecule with steroid structure, acidic in nature, is conventionallybio synthesized from cholesterol in liver [1]. The material has beenunder intensive research in the field of pathology and pharmacology for long years. Of late, it has attracted the attention of material scientists also due to their potential application as building block in designing chiral templates [2], supramolecular structure in soft material [3-5], cation and anion receptors [6-7], artificial ion channel [8] and as surfactant in nanotechnology [9].Bile acids include a group of molecules with similar but non-identical chemical structure, with surprisingly different bio-physical properties. Conventionally,they have24 carbon atoms (abbreviated $\mathrm{C}_{24)}$ with presence of 'non-equivalent hydroxyl group' (in 3, 7 and/or $12 \mathrm{C}$ ) and the side chain structure supporting a carboxylic acid group; which attribute to its peculiar physical and chemical properties [10]. These hydroxyl groups form the hydrophilic face with the carboxylic group of the side chain and also forms an "inner cavity" while the outer face is hydrophobic because of the three methyl groups present there. The carboxyl and hydroxyl group can be easily modified to attach other functional groups.So various kinds of polymers can be prepared with bile acid as pendent group in the main chain and as star shaped polymer with the bile acid in the core. However, synthesis of high molecular weight polymer with bile acid in the main chain is still a challenge [11]. Ahlheim and Hallensleben used ptolunesulfonic acid catalyst and high reaction temperature to obtain polymer with bile acid in the main chain [12]. The principal bile acids are cholic acid, chenodeoxycholic acid, glychocholic acid, taurocholic acid, deoxycholic acid and lithocholic acid. In the present study, we have prepared a polypyrrole composite withcholic acid using DBSA as an additional dopant and ferricchloride as oxidant in aqueous mediumby means of invitro chemical polymerization of pyrrole (Py). The surface morphology of the composite is studied by scanning electron microscope (SEM) while fourier transform infra red (FTIR) spectroscopy givesthe molecular bonding of the monomer and the dopant with the bio-molecule. The composite is exposed to ethanol vapour with different exposure timesand its sensing behavior is studied by impedance spectroscopy at low frequency and also by estimation of the change in its resistance.

\section{MATERIALS AND METHOD}

Pyrrole (Py), cholic acid (CA), hydrochloric acid ( $\mathrm{HCl})$ and dodecyl benzenesulphonic acid (DBSA) are obtained from Fisher Scientific while ferric chloride $\left(\mathrm{FeCl}_{3}\right)$ is obtained from Williams Lab, London. All the materials are analytical grade with very high purity (99.9\%) except Py which is double distilled under reduced pressure before its use. Deionized water is used as medium all through. Chemical oxidative polymerization of $\mathrm{Py}$ is achieved in presence of DBSA where CA is incorporated as a bio-molecule.

\section{EXPERIMENTAL}

\subsection{Preparation of the PPy-cholic Acid Composite}

CAis incorporated on PPy by in-situ co-polymerization of pyrrole. In particular, Py is taken in an aqueous medium 
which is subjected to sonication for 30 minutes. CA is taken in equal weight proportion with de-ionized water and added to the Py solution. Three different samples are made in three different molar proportions of Py and CA, viz. 1:1, 10:1 and 100:1 M/M. Aqueous solution of $\mathrm{FeCl}_{3}$ is added drop-wise in the Py-CA mixture and the resultant solution is subjected to magnetic stirring for two different temperatures : one at room temperature and the other at $0^{\circ} \mathrm{C}$.Each sample is subjected to magnetic stirring for 90 minutes. The solution turns greenish black immediately after addition of $\mathrm{FeCl}_{3}$. The composite obtained as greenish black precipitate is filtered and repeatedly washed by de-ionized water and methanol to remove any oligomer.It is then dried and stored under vacuum. A solution of the composite is used for making thin film by spin casting. Various characteristics of the composite are studied by either using their thin films or using pallets made from the powder sample.

\subsection{Ethanol Sensing}

Ethanol sensitivity measurements are done by monitoring impedance response (Z) and current-voltage (I-V) characteristics of the polymer in presence of ethanol vapour, under $\mathrm{dc}$ and ac bias. A detector is made by pressing the powdered pallet between two steel plates of cross section 1 $\mathrm{cm}^{2}$ with holes in the middle. End of the stainless steel plates are connected to the source meter or the impedance analyzer according to the biasingcondition. The whole system is placed in a $100 \mathrm{cc}$ glass vessel kept in a desiccator.

Characterization is done by means of SEM and FTIR.Electrical properties are studied by measuring currentvoltage $(\mathrm{I}-\mathrm{V})$ characteristics in the voltage range of 0 to 1.0 V. Configuration of electrode attachment is that of a two probe method. Impedance measurement is undertaken at room temperature in the frequency range $50-500 \mathrm{~Hz}$.

\section{RESULTS AND DISCUSSION}

\subsection{Surface Morphology (SEM)}

The effect of DBSA incorporation in PPy and the composite film is observed in the SEM image as shown in Fig 1. Fig $1(\mathrm{~A})$ is the micrographfor PPy which showsaggregation of the particles while Fig.1 (B) represents that of the composite with CA. Considerable change in surface morphology is observed in Fig. 1(B)as the aggregation observed in Fig. 1(A) vanishes there.
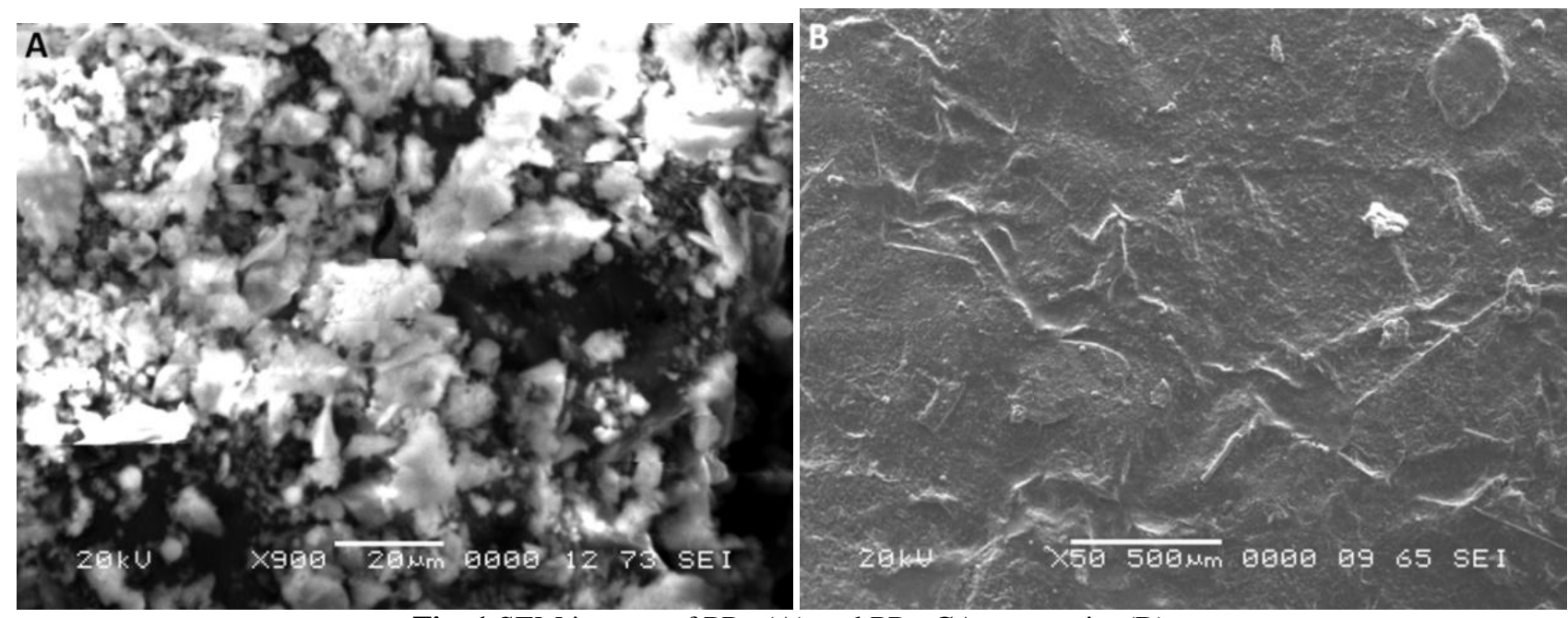

Fig. 1 SEM images of PPy (A) and PPy-CA composite (B)

In the PPy-CA composite shown in Fig. 1(B), the morphology is found to be converted into a layered structure, creating multiple layers with certaincavitiesin PPY-CA which is completely different from the granular aggregates of PPy shown in Fig.1 (A). It is possibly be due to the surfactant effect of $\mathrm{CA}$ which results in multilayer formation because of interacting molecular and electrostatic forces [13].

\subsection{FTIR Spectra}

The FTIR spectra of the samples are shown in Fig. 2 for the DBSA doped PPy and the CA incorporated composite. In PPy, the peak at $2919 \mathrm{~cm}^{-1}$ corresponds to $\mathrm{S}=\mathrm{O}$ and at $2376 \mathrm{~cm}^{-1}$ corresponds to $\mathrm{C}-\mathrm{H}$ stretching vibrationwhich indicates the presence of benzenoid ring of DBSA. 


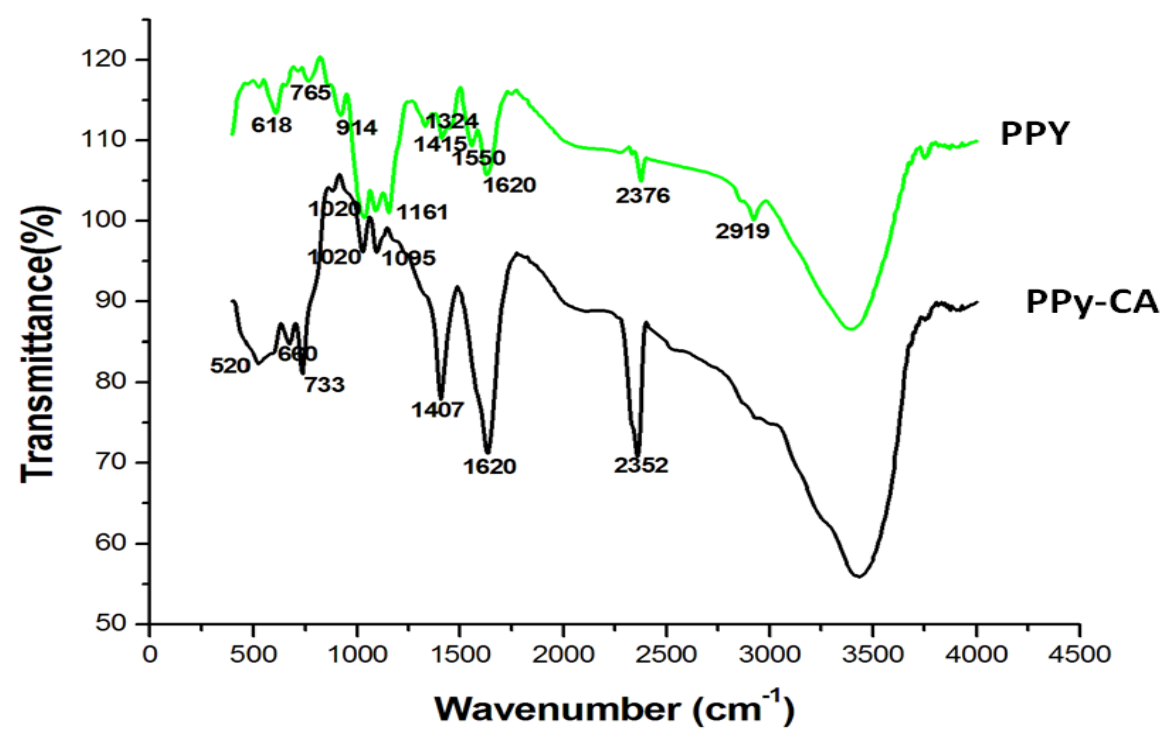

Fig. 2: FTIR spectra of PPy and PPy-CA composite

The peaks at $1620 \mathrm{~cm}^{-1}, 1555 \mathrm{~cm}^{-1}$ and $1415 \mathrm{~cm}^{-1}$ are associated with $\mathrm{C}-\mathrm{N}$ and $\mathrm{C}-\mathrm{C}$ asymmetric and symmetric ring stretching vibrations respectively. The peak at $1085 \mathrm{~cm}^{-1}$ and $1038 \mathrm{~cm}^{-1}$ are attributed to $\mathrm{C}-\mathrm{H}$ deformation and $\mathrm{N}-\mathrm{H}$ stretching vibration. The peak at $1150 \mathrm{~cm}^{-1}$ represents the $\mathrm{S}=\mathrm{O}$ stretching vibration of sulfonate anions $\left(-\mathrm{SO}_{3}\right)^{2-}$ which compensate the positive charge in the PPy chain [14]. The broad peaks around $1300 \mathrm{~cm}^{-1}$ represents the $\mathrm{C}-\mathrm{H}$ and $\mathrm{C}-\mathrm{N}$ in plane deformation. The characteristic PPy peaks are found in the lower wavelength region in which the peak at around $600 \mathrm{~cm}^{-1}$ represents $\mathrm{C}-\mathrm{S}$ stretching due to sulfonate doping [15].

In the CA doped composite the sharp peak appearing at $2352 \mathrm{~cm}^{-1}$ is due to carboxylic group in CA. CA incorporation results in shifting and merging of PPy peaks in the range $1020-1100 \mathrm{~cm}^{-1}$ where the previous peaks merge giving rise to two less intense but sharp peaks at $1020 \mathrm{~cm}^{-1}$ and $1095 \mathrm{~cm}^{-1}$, which are attributed to $\mathrm{C}=\mathrm{O}$ and $\mathrm{C}-\mathrm{H}$ stretching [16] respectively. The characteristic PPy peaks at lower wavelength also merges to give lesser number of vibrational stretching modes, which indicates the change of chemical bonding in the composite. Incorporation of CA might result in certain complimentary bonds stronger then the covalent bond discussed later.

\subsection{I-V Characteristics}

The d-c I-V characteristics for PPy and PPy composite in the voltage range from 0 to $1.0 \mathrm{~V}$ is

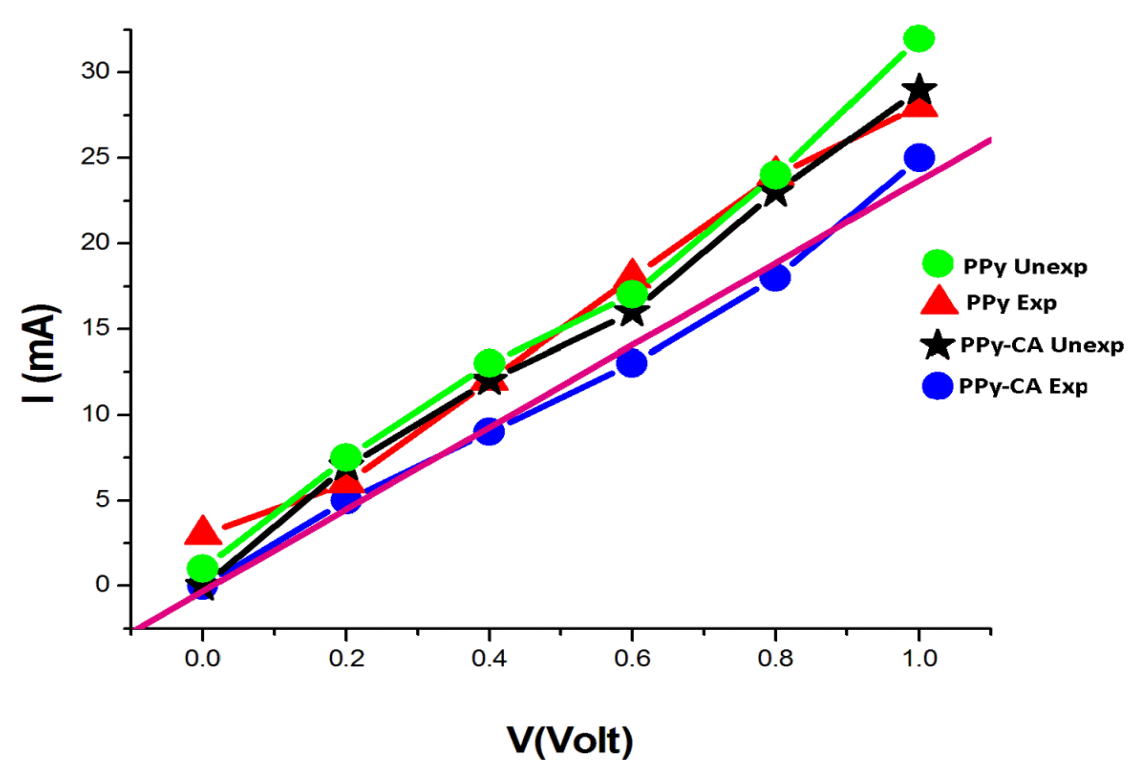

Fig. 3 I-V characteristics for DBSA doped PPy and PPy-cholic acid composite 
shown in Fig. 3 which shows increased slope i.e. decreased resistance, on ethanol intake for both the materials -- PPy and the PPy-CA composite.Resistance of PPy in unexposed state is $3.03 \times 10^{3} \mathrm{Ohm}$ which changes to $0.71 \times 10^{3} \mathrm{Ohm}$, while the resistance of the polymer composite changes from $2.3 \times 10^{3}$ Ohm to $0.39 \times 10^{3}$ Ohm. Though the same order change in resistance is observed in both PPy and its composite, the resistance of the composite is less than that of PPy which facilitates electronic mobility in both unexposed and exposed states.

\subsection{Impedance Measurement and Low Frequency I-}

\section{Characteristics}

The Nyquist plots for pure PPy and the PPy /CA composite for an applied bias of $2 \mathrm{~V}$ is plotted in Fig.4. In the spectrum of the composite an almost vertical line appears in the lower frequencies indicating that the composite behaves like a simple capacitor which can be modeled with a finite transmission line circuit $[17,18]$

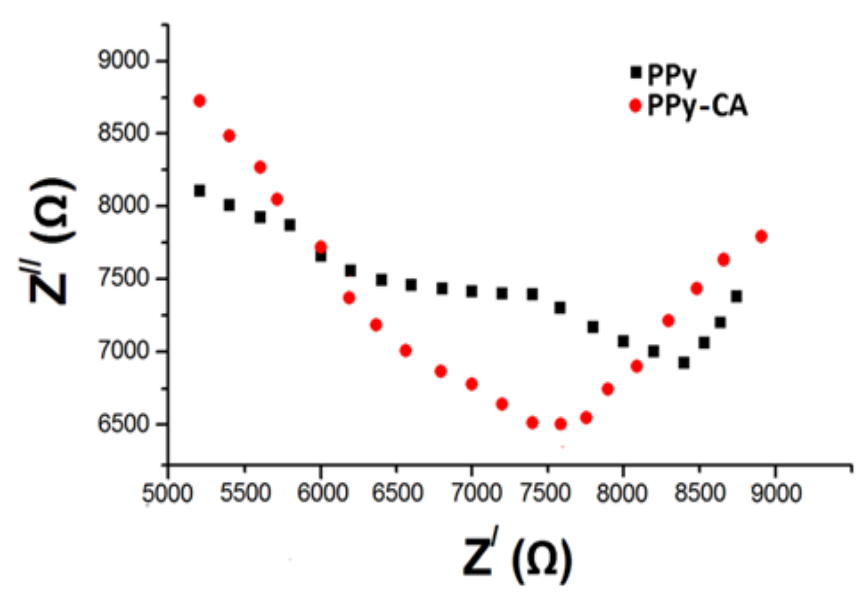

Fig 4 Nyquist plots for pure PPy and the PPy /CA composite

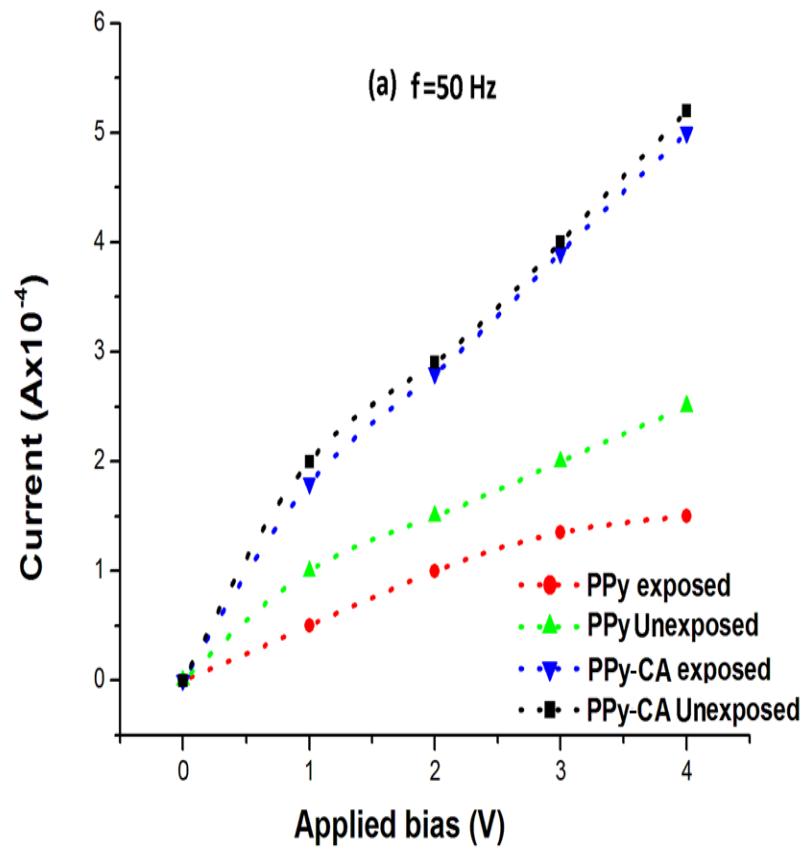

The ionic resistance of the material can be obtained from,

$$
\mathrm{R}_{\text {ion }}=3\left(\mathrm{R}_{\text {low }}-\mathrm{R}_{\mathrm{s}}\right)
$$

Where $\mathrm{R}_{\text {low }}$ is the real axis intercept of the vertical line at low frequency region of the spectra and $R_{s}$ is the real axis intercept in that frequency range.

In contrast to pure PPy,its nano-composite shows no marked transition in the low frequency region. It was reported earlier that addition of the nano-particles leads to catalytic effect and these form an electronic pathway for the polymerized composite [19]. Further, surface adsorption of nanoparticles inhibits one dimensional growth of PPy. This is evident from the morphology of the composite as shown in Fig.1 (B), showing thecompact morphology of the composite.Basically for this reason, low frequency range is the selected range of applied frequency for ethanol sensing study.

\subsection{I-V Measurement in the Low Frequency}

The sensitivity of the polymer composite towards ethanol vapour is tested by recordingthe room temperature change in I-V with applied bias range of 0-4 Volt for different applied frequencies. The I-V characteristics for four different frequencies $(50,100,200$ and $500 \mathrm{~Hz})$ with applied voltage 0 $4 \mathrm{~V}$ are shown in Fig.5. Maximum current is observed in the lowest frequency $(50 \mathrm{~Hz})$ which gradually decreased with increasing frequency. I-V shows a linear trend upto applied voltage of $1.0 \mathrm{~V}$ in all the frequencies, while that trend gradually disappears in higher frequencies. However, in the composite a linear pattern is observed in the entire range of applied bias at $50 \mathrm{~Hz}$; the transition frequency for changeover to non linear nature in both the ethanol treated and untreated samples. The current response is minimum at $500 \mathrm{~Hz}$. The result shows that impedance is increasing with frequency for the particular applied bias range.

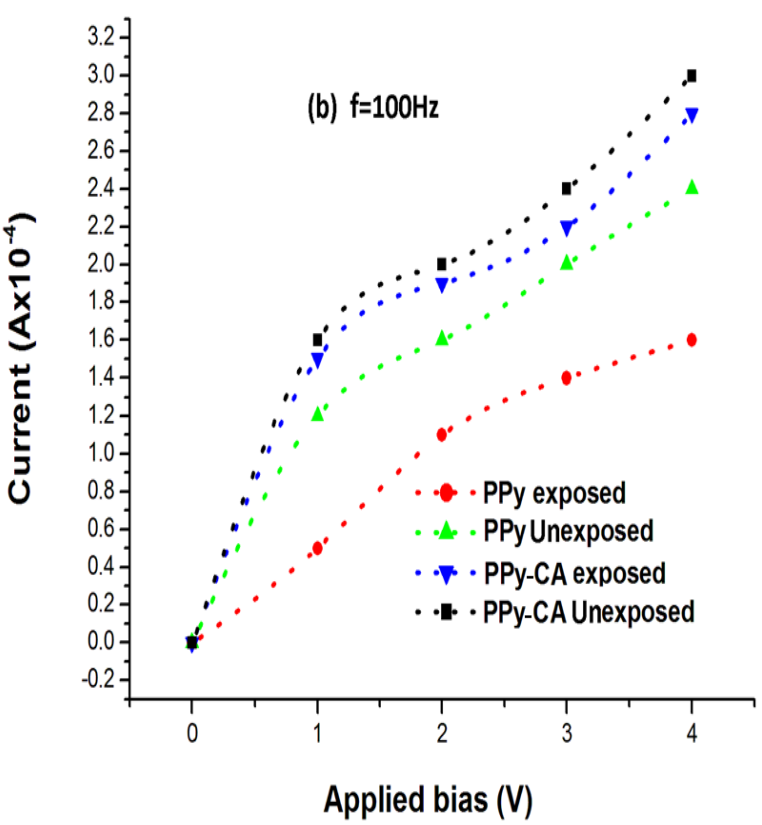



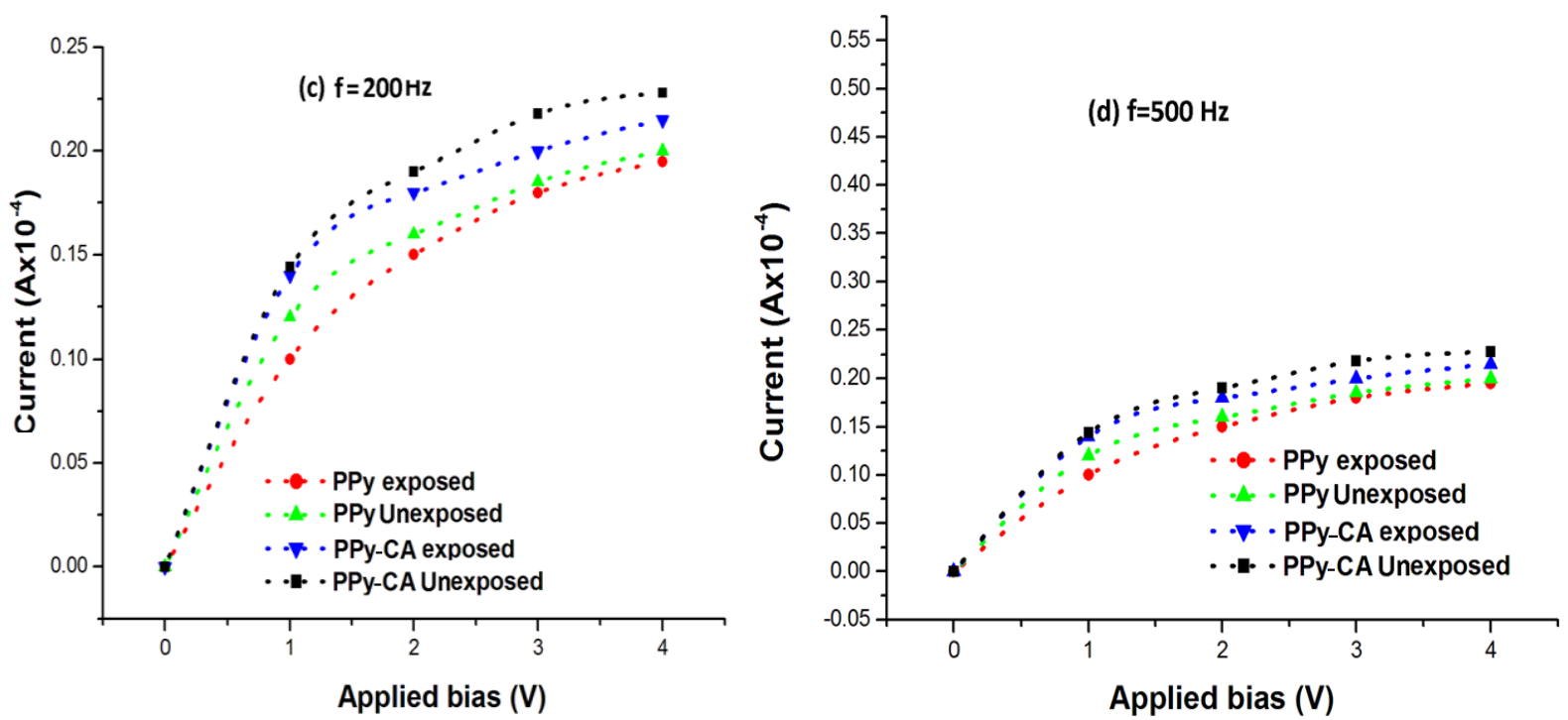

Fig.5 I-V characteristics of PPy and PPy/CA composite for applied frequencies of (a) $50 \mathrm{~Hz}$ (b) $100 \mathrm{~Hz}$ (c) $200 \mathrm{~Hz}$ and (d) 500 $\mathrm{Hz}$

\subsubsection{Impedance Measurements: Ethanol}

\section{Sensitivity in Terms of Impedance}

changes with time at aparticular bias voltage, with respect to ethanol incorporation. The results are shown in Fig. 6.

Along with I-V characteristics, we have also observed how the impedance response $(\mathrm{Z})$ of PPy and the composite
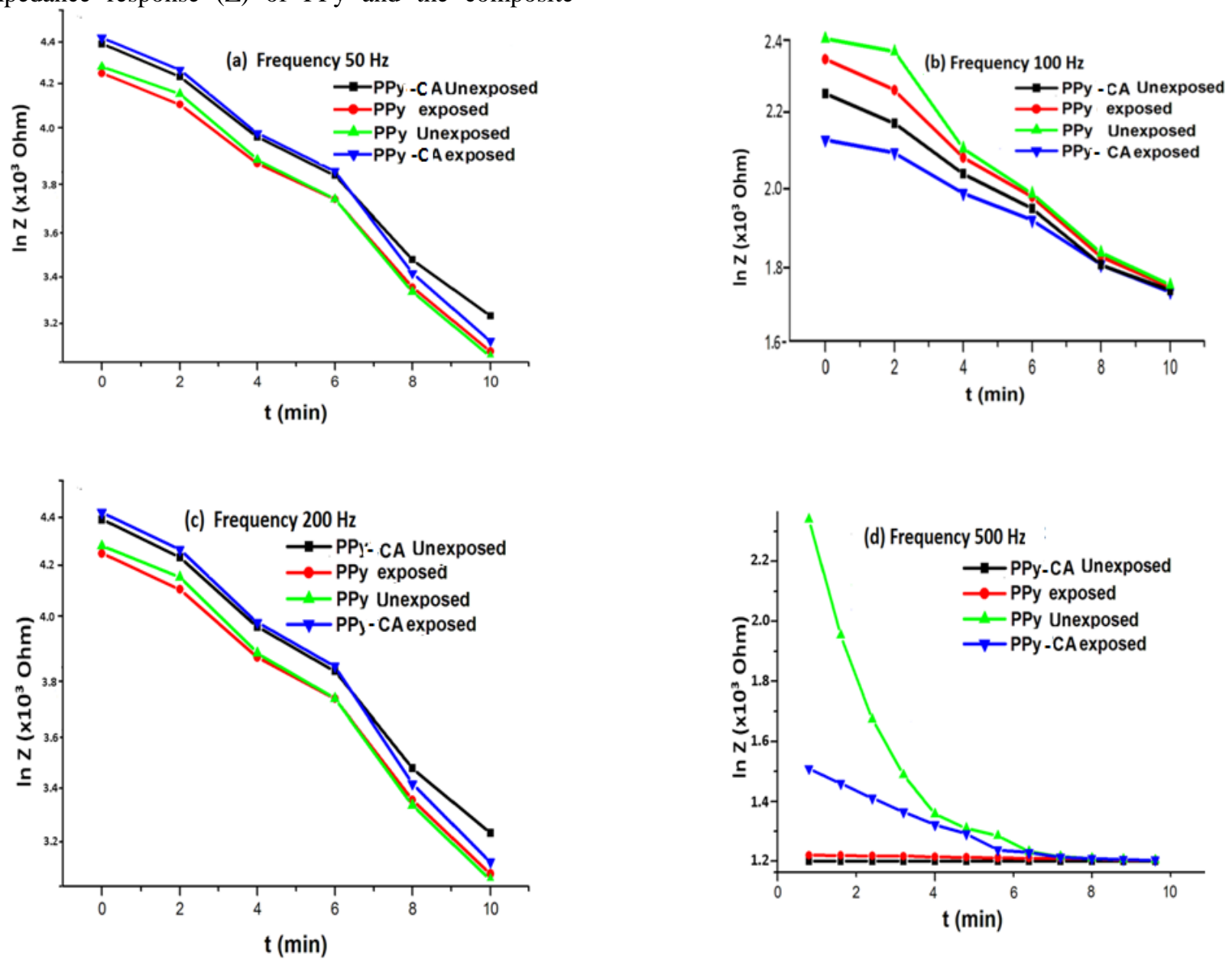

Fig. 6 Impedance vs time in four different applied frequencies (a) $50 \mathrm{~Hz}$ (b) $100 \mathrm{~Hz}$ (c) $200 \mathrm{~Hz}$ and (d) $500 \mathrm{~Hz}$ 
The figure shows decrease in $\mathrm{Z}$ with time as well as with frequency while a quite different nature is observed at applied frequency of $500 \mathrm{~Hz}$.

\section{THEORY OF SENSING}

\subsection{Physical: Adsorption}

Conducting polymer(CP)s interact with bio-molecules basically through intermolecular and surface forces acting as mediators. These forces are broadly Van der Waals, electrostatic, hydrophobic and hydrogen bonding [20]types and typically classified as Lifshitz Van der Waals (which is non polar, dispersive in nature) and Lewis acid base (which is polar in nature)force. Interaction of bio-molecule generally occurs immediately after its initial exposure in the material-liquid interface. Major limitation in the process is non-specific binding of the host molecule which determines selectivity needed in biosensor or actuator applications [21]. Specific interactions arise from a unique combination of forces that act in a directional manner forming, for instance, complimentary bonds [22] which may be stronger than the covalent bond existing between the $\mathrm{CP}$ and the dopant molecule. Though a lot of work has been done in the field of $\mathrm{CP}$ biosensor, very little information is found in literature which can give a quantifying model of the intermolecular and surface forces [23-25]. Thus one has ample scope to analyze the adsorption and sensing behavior of the PPy composite.

According to Langmuir's adsorption theory, the gas molecules impinging on any solid surface do not generally rebounce but condense on the surface, being held by the field of force of the surface atoms and eventually evaporate from the surface. The time lag for evaporation depends on the intensity of the surface forces. Adsorption is the direct result of this time lag. The range of these surface forces is $10^{-10} \mathrm{~m}$ (i.e. the dimension of a molecule)[26]. Thus with the decreasing size of the molecules the dimension of the surface forces increases which can hold the molecules in the surface. This happens in the present case when the PPy-CA composite is exposed to ethanol vapour. The oxygen from the $\mathrm{OH}$ groupin ethanol gets attached to the hydrophilic sites of CA forming the "inner cavities" in the polymer composite which undergo an ionic transformation leading to enhanced conductivity as shown in Fig.3 and 5. Selective adsorption of the bio-molecular composite is influenced by intermolecular and other competing forces which may result in decrease in interfacial interaction energy $\left(\mathrm{E}_{\mathrm{int}}\right)$. Interfacial interaction plays a vital role in case of any bio-molecular incorporation in a $\mathrm{CP}$ and hence selective adsorption is observed when the CA incorporated PPy is exposed to ethanol vapour. It has been hypothesized that such interactions induces an alteration in the double helix of the polymer structure and subsequently allows formation of hydrogen bonds and intercalation of the polymer with formation of $\mathrm{N}-\mathrm{H}$ bond as well as N-H- $\pi$ interaction[27] after exposure to ethanol vapor, resulting in subsequent change in conductivity as exhibited by the I-V characteristics.

\subsection{Chemical}

The adsorbed oxygen molecules on composite surface forms ionic species $\mathrm{O}^{-}, \mathrm{O}^{2-}$ and $\mathrm{O}_{2}$ - which acquire electrons from the PPy surface; PPy being a good electron donor with delocalized electrons[28]. The polymer blend also undergoes a reaction on exposure to ethanol vapour. Such an interaction, which is non-covalent in nature, takes place in between the aromatic rings of the polymer blend and the ionic species of oxygen $\left(\mathrm{O}^{-}, \mathrm{O}^{2-}\right.$ and $\left.\mathrm{O}_{2}^{-}\right)$which was studied by Germain et al and introduced the term ' $\pi$-stacking' [29].Electrons participating in such interactions are free to migrate into the polymer surface and results in its increased conductivity.

The reaction kinematics may be expressed as [30],

$$
\begin{gathered}
\mathrm{O}_{2} \text { (gas) } \Leftrightarrow \mathrm{O}_{2} \text { (adsorbed) } \\
\mathrm{O}_{2} \text { (adsorbed) }+\mathrm{e}^{-}<=>\mathrm{O}_{2}^{-} \\
\mathrm{O}_{2}^{-}+\mathrm{e}^{-}<=>2 \mathrm{O}^{-}
\end{gathered}
$$

The reaction between ethanol and ionic oxygen can take place in two different ways:

$$
\begin{aligned}
& \mathrm{C}_{2} \mathrm{H}_{5} \mathrm{OH} \text { (gas) }+\mathrm{O}^{-} \Leftrightarrow>\mathrm{CH}_{3} \mathrm{CHO}+\mathrm{H}_{2} \mathrm{O}+\mathrm{e}^{-} \\
& \mathrm{C}_{2} \mathrm{H}_{5} \mathrm{OH} \text { (gas) } \Leftrightarrow>\mathrm{H}+\mathrm{C}_{2} \mathrm{H}_{5} \mathrm{O} \text { (surface) } \\
& \mathrm{C}_{2} \mathrm{H}_{5} \mathrm{O} \Leftrightarrow=\mathrm{H}+\mathrm{CH}_{3} \mathrm{CHO} \\
& \mathrm{CH}_{3} \mathrm{CHO}+\mathrm{O} \text { (bulk) } \rightarrow \mathrm{CH}_{3} \mathrm{COOH}+\mathrm{O} \text { (vacancies) }
\end{aligned}
$$

The conductivity arises from electron transfer along the conjugated $\pi$-molecular backbone coupled with the motion of the charge carriers after exposure to ethanol vapour. On oxidation, an electron is removed from the $\pi$ system producing a cation which constitutes a polaron when associated with the local distortion. Thus a polaron is formed in every four pyrrole unit. On further oxidation, pairs of polarons combine to form bipolarons which are energetically more favourable. Bipolarons can migrate along the polymer chain providing the charge transport in the PPy structure [31]. The conductivity is however determined by the charge transfer between the dopant and the remaining part of the polymer segment: charge carrier mobility within a single polymer chain and charge transfer (i.e. hopping) between different polymer chains [32]. The exposure to ethanol enhances the charge carrier mobility in the $\mathrm{CP}$ which is further stimulated by incorporation of $\mathrm{CA}$ in the polymer [33].

\section{CONCLUSION}

Incorporation of CA on DBSA doped PPy results in the formation of a polymer composite which has supramolecular structure with imprinted properties of PPy as well as CA. Certain properties are modified with resultant change in topography and electronic conductivity of the composite, 
probably due to formation of certain complimentary bonds stronger than the covalent bonds caused by interaction of the surface forces when CA is incorporated in the polymer. Change in conductivity is due to enhanced surface charges formed with the incorporation of the bio-molecule. The change is prominent when the composite is exposed to ethanol vapour, due to protonation of the oxygen molecules in the $\mathrm{OH}$ group of ethanol, adsorbed in the surface cavities of the composite. Both resistance and impedance of the of the polymer composite show characteristic changes on ethanol exposure which is stronger in the lower frequency range. The change in impedance is most prominently observed in $50 \mathrm{~Hz}$ frequency when the current is found maximum and linearity is observed in low frequency range with applied bias range of 0 to 1 Volt. With increasing frequency the $\mathrm{I}-\mathrm{V}$ curves shows gradual decrease in the value of current for the same applied bias indicating increased value of impedance, which is normal in case of CP's like PPy. AC conductivity increases with increasing electronic mobility till a saturation level is reached and inter chain hopping continues, which is stimulated by the frequency of the applied field. Cholic acid incorporation possibly causes surface modification of the polymer which facilitates electronic mobility and on exposure to an ionizing vapour (e.g. ethyl alcohol); the saturation level is reached at a very low frequency.

\section{REFERENCES}

[1] M. Axelson,E. Ellis, B. Mörk, K. Garmark , A. Abrahamsson, I. Björkhem, B. G. Ericzon andC. Einarsson. Hepatology.31, 2000, 1305.

[2] AK Bandyopadhyaya, N. M. Sangeetha and U. J. Maitra.Org Chem. 65,2000, 8239.

[3] V. H. Soto Tellini, A. Jover, L. Galantini, N.V. Pavel, F. Meijide and J. Vázquez Tato.J Phys Chem B. 110,2006, 13679

[4] V.H.Soto Tellini, A. Jover, F. Meijide, J. Vázquez Tato, L. Galantini and N.V. Pavel. Adv Mater. 19, 2007, 1752.

[5] P. Babu, N. M. Sangeetha and U. Maitra. Macromol Symp. 241, 2006, 60.

A. P. Davis and J. B. Joos. Coord Chem Rev. 240,2003, 143.

[6] S. Ghosh, A. R. Choudhury, T. N. Guru Rowand U. Maitra.Org Lett. 7,2005, 1441.

[7] M. Yoshii, M. Yamamura, A. Satakeand Y. Kobuke.Org Biomol Chem. 2, 2004, 2619.

[8] M. Alvarez Alcalde, A. Jover, F. Meijide, L. Galantini, N.V. Pavel, A. Anteloand J. Vázquez Tato.Langmuir. 24,2008, 6060.

[9] X. X. Zhuand M. Nichifor.Acc Chem Res, 35, 2002, 539.

[10] X. Z. Hu, Z. Zhang, X. Zhang, Z Y Li, Z.X. Xhu. Steroids, 2005, 70: 531.

[11] M.Ahlheim, M. L. Hallensleben. Makromol Chem, Rapid Commun, 1988, 9: 299.

[12] P.P D. Sharma and D. Sarkar.Ind J Biochem Biophys 52 (2), 2015, 203.
[13] R.M.Silverstein, G.C. Bassler, T. C. Morrill. Spectroscopicidentification of organic compounds. 5th edn, Wiley, 1992

[14] The Aldrich Library of FTIR spectra, 1997

[15] A.Benrebouh,D Avoce and X. X Zhu. Polymer 42, 2001, 4031.

[16] G. Garcia-Belmonte, J. Bisquert, Electrochim. Acta, 47,2002, 4263

[17] X. Ren and G. Pickup.J. Phys. Chem., 97, 1993, 5356

[18] Y.C. Liuand C.J. Tsai, J. Electroanal. Chem., 537, 2002,165

[19] D. Leckbandand J.Israelachvili, Quart. Rev. Biophys. 34,2001, 105.

[20] M. Gerard, A. Chaubeyand B.D. Malhotra, Biosens. Bioelectron. 17, 2002, 345.

[21] X .Yang, C.O. Too, L. Sparrow, J. Ramshaw and G.G. Wallace, React. Func. Polym. 53, 2002, 53.

[22] X. Cui, V.A.Lee, Y. Raphael. J.A Wiler, J. F. Hetke, D. J AndersonandD. C Martin J. Biomed. Mater. Res. 56, 2001,261.

[23] C.Saltó, E Saindon, M.Bolin, A.Kanciurzewska, M.Fahlman,E. W Jager and P Tengvall.Langmuir, 24,2008, 14133.

[24] S CosnierandM. Holzinger, Chem. Soc. Rev. 2011, 40, 2146.

[25] J Preat, B.Teixeira-Dias, C.Michaux, E. A Perpèteand C. Alemán, J. Phys. Chem. A, 2011, 115, 13642.

[26] J. Preat, D. Zanuy, E. A Perpèteand C. Alemán, Biomacromolecules, 201,12, 1298.

[27] L. Misevic ¿iene', Ž . Anusevi`ciusand J. N. Sarlauskas, Acta Biochimica Polinica 53, 2006, 569.

[28] M.E. Germain and M.J. Knapp, Journal of the American Chemical Society 130, 2008, 5422.

[29] H F Hellegourac,F Aefi Khonsari, PR lanadeandandJ. Amourax. Sens Actuat B, 73, 2001, 27.

[30] G.Inzelt, M.Pineri, J. W. Schultze, and M. A.Vorotyntsev, Electrochim. Acta 45, 2000, 2403.

[31] A.Bhattacharya, A. DeandS. Das, Polymer 34,1996, 4375 . 